\title{
Tecnología Anticonceptiva: Presente y Futuro
}

\author{
ALFREDO GOLDSMITH, MD, MPH \\ DAYID A. EDELMAN, PhD
}

\section{INTRODUCCION}

Durante los últimos veinte años se han efectuado un número significativo de avances en métodos de control de la fecundidad. Antes de la mitad de la década de los años cincuenta, no existían métodos anticpnceptivos efectivos que no fuesen esterilización masculina o femenina, pero cerca del final de los años cincuenta, como resultado de los trabajos de Rock, Pincus y García, las progestinas y los estrógenos administrados oralmente demostraron ser anticonceptivos eficaces. El desarrollo de anticonceptivos orales ha sido el avance más significativo en tecnología anticonceptiva. El método es barato, fácil de administrar y efectivo. Después del desarrollo y de la distribución de anticonceptivos orales durante los últimos quince años, no solamente ha continuado la investigación en anticonceptivos sistemáticos, incluyendo aquellos que no requieren administración diaria, sino que ha habido también un mayor avance en otros métodos de anticoncepción. En la década de los sesenta, el desarrollo del dispositivo Lippes Loop renovó el interés en anticoncepción intrauterina, y desde ese tiempo muchas formas, tamaños, y materiales han sido investigados para dispositivos intrauterinos. Mientras que muchos de los dispositivos intrauterinos nunca progresaron más allá de la etapa de desarrollo como resultado de efectos adversos tales co- $^{-}$ mo alta tasa de embarazo, expulsión y retir , o dificultades en inserción, otros disp sitivos han demostrado ser eficaces anticonceptivos.

Antes del desarrollo de las técnicas de endoscopia para esterilización femenina en

Deputy Head Design and Analysis Division International Fertility Research Program Research Triangle Park, North Carolina.

Area Coordinator for Latina America International Fertility Research Program Research Triangle Park, North Carolina la decada del sesenta, la esterilización femenina en la déca

menina permanente era generalmente $\mathrm{e}$ fectuada bajo anestesia general y necesita ba tres o cinco dias de hospitalización. Muchos de los métodos de esterilización femenina que están actualmente en uso. tales como laparoscopía, culdoscopía y minilaparotomia, son efectuados en forma ambulatoria bajo un mínimo de anestesia y permiten a la mayoria de las pacientes reasumir sus actividades normales en un corto período de tiempo. Más aún, la efectividad de este procedimiento ha sido aumentada por el desarrollo de equipos y técnicas mejores de oclusión tubaria.

Todos los métodos de anticoncepción están asociados con fracaso. A pesar que en teoría algunos métodos tienen una tasa de cero, como la esterilización y los anticonceptivos orales, bajo ciertas condiciones de uso, las tasas de fracaso son mucho másaltas (Cuadro I). Falla de tomar el anticonceptivo oral de acuerdo con los actuales regimenes de prescripcion, ligadura de estructuras distintas de las trompas. reanostomosis de los vas deferens y de los tubos de Falopio, etcétera, contribuyen a la tasa de fracaso de los anticonceptivos y de la esterilización.

En este reporte serán reseñados algunos de los avances recientes en tecnologia ais: ticonceptiva. Este resumen ha sido limitado a avances en anticoncepción intraterina, anticoncepción oral, esterilización femenina y aborto. A pesar de que aborto per se no es considerado como método de anticoncepción, es, sin embargo, un método efectivo de control de la fecundidad. El énfasis en este reporte va dirigido hacia el desarrollo de nuevos métodos que parecen ofrecer ventajas en términos de eficacia $y$ de menores complicaciones.

\section{METODOS ANTICONCEPTIVOS}

Dispositivos Intrauterinos (DIUs). 
A pesar de que los dispositivos intrauterinos pueden ser insertados en cualquier $\mathrm{e}^{-}$ tapa del ciclo menstrual, después de un aborto inducido o espontáneo, o después de un parto de término, todos los tipos de dispositivos intrauterinos están asociados con efectos adversos y complicaciones. Muchos de los dispositivos intrauterinos son efectivos para uso clínico, pero con todos ocurren algunos embarazos con el todos ocurren algunos embarazos con el dispositivo in situ.

Se ha demostrado en estudios que la eficacìa de un dispositivo intrauterino está en parte relacionada con su área de superficie, su espesor y su flexibilidad. Al aumentar el área de superficie de un dispositivo intrauterino parece existir una co- $^{-}$ rrelación con la disminución de tasas de embarazo. Sin embargo, usando dispositivos con superficie grande, aumenta el número de episodios y la duración de sangrado intermenstrual.

Los dispositivos que contienen cobre o los que contienen drogas no parecen tener ninguna ventaja significativa sobre los dispositivos inertes, (dispositivos manufacturados de polietileno o de acero inoxidable). El efecto del cobre en algunos tipos de dispositivos parece ser benéfico en relación con la reducción de tasas de embarazo con el dispositivo in situ. En las investigaciones iniciales del dispositivo $T$, la tasa de embarazo al año fue aproximadamente de 18 por cada cién usuarias. Con la adición de $200 \mathrm{~mm}^{2}$ de cobre se produ ce una reducción en la tasa de embarazo a 1-3 por cada cién mujeres. Sin embargo, con el Lippes Loop C, un dispositivo que tiene un área de superficie mayor que el dispositivo $\mathrm{T}$, no hubo ninguna disminución significante en la tasa de embarazo cuando se le añadieron $200 \mathrm{~mm}^{2}$ de cobre al dispositivo. El efecto de la adición de otros metales como acero, plata y zinc a los dispositivos está siendo evaluado en este momento. En el presente no existe evidencia que indique que la adición de tales metales podrá mejorar significativamente la eficacia de los dispositivos intrauterinos.

Todavía no hay evidencia conclusiva que muestre que la adición de agentes antifibrinolíticos, (tales como ácido tranexamico) y progesterona a algunos dispositivos tendrá una ventaja sígnificativa sobre los dispositivos inertes o sobre aquellos que contienen cobre. Los resultados de estudios de dispositivo Progestasert que descarga $65 \mathrm{mcg}$ de progesterona al día y del Spring-Coil IUD con un agregado de progesterona $(60.0 \mathrm{mg})$ no han indicado ningún beneficio importante al ser comparados con los dispositivos inertes (Cuadro II). En un estudio de Spring-Coil IUD, el agregar ácido tranexámico al dispositivo de silástico muestra que la adición de este agente produce una reducción en el número de episodios de sangramiento intermenstrual. Sin embargo, el Spring-Coil es un dispositivo de superficie grande, $1755 \mathrm{~mm}^{2}$, y está asociado con alta tasa de retiro por hemorragia y también con alta tasa de embarazos.

A pesar que existe la tecnología para evaluar dispositivos intrauterinos, la comparación de los resultados de distintos investigadores resulta muy difícil debido a que resultados disímiles con el mismo dispositivo han sido reportados por diferentes investigadores (Cuadro II). Estas diferencias pueden ser en parte debidas a diferentes criterios usados para evaluar el dispositivo y a diferencias en la población estudiada, en las técnicas de inserción y en el tiempo de la inserción.

Los dispositivos requieren una extensiva evaluación para asegurar que son seguros para uso clínico y no simplemente asociados con tasas bajas de embarazo, expulsión y retiro. El DIU Dalkon Shield, según algunos estudios clínicos, está asociado con tasas bajas de embarazo, expulsión y retiro. Sin embargo en 1974, como resultado de una extensiva investigación de este dispositivo, se observó que este dispositivo, al compararse con otros estaba asociado con una tasa mayor de abortos sépticos espontáneos durante el segundo trimestre de embarazo con el dispositivo insitu. Se emitió la teoría de que las altas tasas de abortos sépticos espontáneos fueron debidas al tipo de cola multifilamental, que actuaba como un medio para que las bacterias invadieran el útero. Todos los demás dispositivos intrauterinos actualmente en uso tienen colas monofilamentales. Como resultado de estos eventos el Dalkon Shield ya no es producido por la compañ ía Robbins.

Se requieren más investigaciones para conocer los mecanismos de acción de los

dispositivos. A pesar de que existen nu- 
merosas hipotesis que parcialmente explican el efecto anticonceptivo de los dispositivos, estas hipótesis se basan en hallazgos asociados que pueden o no estar directamente relacionados con los efectos y se basan, la mayoría de las veces, en estudios en animales no siempre aplicables a la raza humana.

El desarrollo de dispositivos más efectivos, tanto inertes como bioactivos dependerá de un mejor entendimiento del mecanismo de los dispositivos intrauterinos, del desarrollo de material apropiado para actuar como conductor de medicamentos, $y$ del uso de medicamentos que tengan una alta y prolongada efectividad contraceptiva.

Anticonceptivos orales.

Los anticonceptivos más comunmente usados son los combinados, para un régimen de 21 dias, que contienen estrógeno y progestágeno. Todos estos regimenes han demostrado efectividad en prevenir embarazos, sin importar el tipo de estrógenos y progestágenos usado. Como resultado de la reportada relación entre el contenido de estrógeno en la píldora y sus eventos tromboembólicos, ha surgido un movimiento tendiente a la reducción del contenido de estrógeno en los anticonceptivos orales en un standard de $0.05 \mathrm{mg}$ a $0.020-0.035 \mathrm{mg}$ por pildora. El problema principal de estas preparaciones con dosis bajas de estrógeno no es de eficacia, sino de que una importante proporción de mujeres que toman anticonceptivos orales con dosis bajas de estrógeno, padecen de sangrado con relativa frecuencia, lo cual es un efecto colateral serio.

A pesar de que los anticonceptivos orales de régimen combinado son los más frecuentemente usados, otros han demostrado también ser efectivos, aunque en menor proporción que los combinados que describimos anteriormente. Las "mini-pill" o pildoras que contienen progesterona solamente -la misma progesterona usada en las preparaciones combinadas (norgestrel, norethindrone) - son efectivas para prevenir embarazos aunque tanto como los anticonceptivos orales combinados. Muchos de los efectos colaterales asociados con el régimen combinado, también lo están con el régimen de progesterona sola o "minipill"; nos referimos a efectos tales como aumento o disminución de la duracion y cantidad del fjujo menstrual, spotting, amenorrea, y ciclos menstruales irregulares.

Los anticonceptivos sistemáticos de efecto prolongado como medroxiprogesterona $150 \mathrm{mg}$ administrada cada 90 días, han sido un método efectivo en la prevención de embarazo, pero en menor grado que las preparaciones combinadas. A pesar de que este método sistémico de larga duración está asociado con tasas bajas de efectos colaterales adversos, cuando se compara con el régimen combinado, su principal desventaja es que está asociado con irregularidades mesntruales que frecuentemente ocurren y que varían desde amenorrea hasta metrorragia.

Existen ciertas ventajas en el uso de anticonceptivos sistémicos que no requieren administración diaria, debido a que una de las más frecuentes causas del fracaso de régimen diario es que las pacientes no toman los anticonceptivos como han sido prescritos.

Otro de los anticonceptivos sistémicos de larga duración que ha demostrado ser efectivo es el Unovis, distribuido por Warner-Lampert, es una preparación de Quinestrol $2.0 \mathrm{mg}$ (estrógeno de fórmula 3ciclopentil éter de etinil estradiol y que tiene un almacenamiento prolongado $y$ subsecuentemente descarga del tejido graso en el cuerpo). El Unovis se adminis tra oralmente cada 28 días. A pesar de que los anticonceptivos de efecto prolongado tales como Depo-Provera y Unovis pueden tener teóricamente mayores tasas de fracaso que los contraceptivos orales, bajo condiciones de práctica, su tasa de fracaso puede ser más bajas en algunas poblaciones.

Se necesitan estudios apropiados para evaluar los efectos y complicaciones a corto y largo plazo de todos los anticonceptivos sistémicos. En particular, tendrán que efectuarse estudios para evaluar los anticonceptivos sistémicos que puedan ser administrados inmediatamente después de un embarazo y que no afecten al lactante o que por el contrario, aumenten la capacidad de lactancia.

\section{Esterilización Femenina}

La mayoría de los métodos quirúrgicos de esterilización femenina permanente, con excepción de histerectomía, han nresentado una tasa aceptable de complica- 
ciones clínicas. Los métodos de esterilización no quirúrgicos, como la instilación transcervical de quinacrina, todavía están en etapa experimental de desarrollo y el uso de estas técnicas se asocia a tasas altas de fracaso. Así mismo, la oclusión de las trompas por histeroscopia, usando tapones y gomas no ha sido todavía aceptada como un procedimiento práctico para uso general. La desventaja principal de la histeroscopía consiste en que en una proporción significativa de las pacientes, el cirujano no puede visualizar adecuadamente las trompas para poderlas ocluir.

$\mathrm{Si}$ el objetivo de preferir un procedimiento de esterilización es el proveer el método más seguro de anticoncepción que requiera una duración mínima de hospitalización, que pueda ser realizado usando un mínimo de anestesia, y que permita a las mujeres regresar a sus actividades normales en un período de tiempo corto, la laparoscopía, la culdoscopía, y la minilaparotomía son aparentemente los procedimientos mejores. No existe un método de esterilización que sea el mejor para todas las clínicas y para todas las pacientes. En cualquier clínica, la decisión de utilizar un método de esterilización sobre otro, depende de la existencia del personal médico. El ideal sería que en cualquier clínica existiera más de un método de es. terilización.

La laparoscopía puede ser realizada como un procedimiento en el post-aborto inmediato (inducido o espontáneo), o en el puerperio inmediato. Varias investigaciones han demostrado que no obstante el momento en que se realiza el procedimiento, la laparoscopía se asocia con tasas minimas de complicaciones operatorias y post-operatorias. Con la laparoscopía, así como con otros métodos de esterilización, se están desarrollando evaluando técnicas mejoradas para la oclusión de las trompas

"Dos de éstas técnicas, muy prometedoras, son la aplicación de "springloaded" clips (figura 1) y de bandas silásticas (figura 2). Los clips se colocan en la porción ampular de la trompa, utilizando un laparoscopio y un aplicador diseñados especialmente (Figuras 3 y 4). Los clips consisten de dos mandíbulas plástocas con un resor te de acero inoxidable que mantiene a las dos mandíbulas cerradas alrededor de la trompa, después que el clip ha sido colocado. Fste clip causa un daño mínimo a la trompa, y aunque todavía no se ha sido intentado en mujeres, se ha obtenido éxito con la reanastomosis de las trompéxito con la reanastomosis de las trompas en animales.

Las bandas silásticas (goma siliconizada), también se colocan a través de un laparoscopio especialmente diseñado (Figura 5). La banda se coloca en la porción ampular de la trompa y produce una oclusión del tipo Madlener (Figura 6). Tanto los springloaded clips las bandas silásticas, pueden ser también colocados después de laparotomía, culdoscopía, y colpotomía. Se han utilizado otros tipos de clips para oclusión; con culdoscopía se han implantado tantalum clips (clips de tantalio). Algunos investigadores han reportado una tasa alta de fracaso con la aplicación de un clip para cada trompa, pero la aplicación de dos clips para cada una, aparentemente produce tasas de fracaso más satisfactorias.

Mientras que la culdosopía se usa extensivamente en algunas áreas del mundo, como México, India, Tailandia y Líbano, è los Estados Unidos y en Europa Occidental no ha obtenido la popularidad de la laparoscopía y en muchos de los hospitales universitarios el procedimiento no se enseña al personal residente. No obstante que la culdoscopía es un procedimiento técnicamente más difícil que la laparoscopía, sigue teniendo un lugar destacado en el arsenal de procedimientos quirúrgicos para esterilización. Al igual que la laparoscopía, la culdoscopía puede ser realizada en pacientes ambulatorias, bajo an estesia minima, usando solamente anestésicos loca nima, usando solamente anestésicos locales. Una de las dificuiltades más frecuentes durante la culdoscopía es la visualización adecuada de las trompas, así como el bajar las trompas al fondo de saco de Douglas para ligarlas. En un estudio de 525 culdoscopías realizadas en tres hospitales en Jamaica, Líbano y Singapur, se reportaron dificultades en un $\mathbf{1 0 . 0}$ por ciento de los procedimientos cuando fueron realizados por cirujanos con experiencia. El desarrollo de los aplicadores para el "spring-loaled" clip y de la banda silástica para uso en culdoscopía podría eliminar muchas de las dificultades técnicas en el momento de la cirugía, ya que al usar estos aplicadores las trompas pueden ser ocluídas en la cavidad peritoneal.

Otros métodos de oclusión mecánica es- 
tán siendo desarrollos y evaluados. Rocket de Londres está probando una modifica ción del "spring-loaded" clip que se aplica por laparoscopía usando la técnica de dos punciones. En Inglaterra, en la Universidad de Nottingham, un clip formado de silástico dentro de tantalio (Figura 7) promete ser efectivo, si nos basamos en los resultados de pruebas precoces con animales.

Probablemente las mayores desventajas de la laparoscopía y la culdoscopía son la experiencia requerida para realizar los pro cedimientos, el costo del equipo y la dificultad encontrada frecuentemente para reemplazar partes del equipo en países en estado de desarrollo. Una alternativa de la laparoscopía y de la culdoscopía es la minilaparotomía, en la cual el acceso a las trompas se obtiene a través de una incisión pequeña suprapública. El procedimiento puede ser realizado por médicos con entrenamiento ginecológico extensivo, bajo anestesia mínima y en pacientes ambulatorias. Con el procedimiento de minilaparotomía las trompas pueden ser ocluídas utilizando clips, bandas silásticas, o cualquiera de las técnicas de ligadura standard.

Aunque las tasas de complicaciones quirúrgicas y post-operatorias precoces de la laparoscopía, la culdoscopía y la minila parotomía, se encuentran en un nivel clínico aceptable, las complicaciones a largo plazo, las tasas de fracaso y la necesidad de cirugía subsecuente para estos procedimientos y sus técnicas asociadas de oclusión de trompas no han sido adecuadamente evaluadas. Se necesitan más estudios e investigaciones al respecto.

\section{Aborto}

Sin tener en cuenta la legalidad del aborto, este es no obstante, un procedimiento utilizado por las mujeres para controlar su fecundidad. Desde principios de 1970 el aborto ha sido legal en varios países incluyendo los Estados Unidos, India, Tailandia, Singapur, e Italia. En los Estados Unidos, como consecuencia de la legalización, se ha observado un aumento en el número de abortos inducidos legalmente, acompañado por una disminución en el número de abortos inducidos ilegalmente. Mientras que el número de abortos inducidos ilegalmente no se conoce, su reducción se observa a través del menor número de muertes atribuídas al aborto de este tipo.

En todos los países latinoamericanos, las consecuencias del aborto ilegal inducido son bien conocidas: muerte, hospitalización prolongada por el tratamiento de complicaciones, sepsis, y probablemente tasas más altas de infertilidad como resultado directo de la sepsis. El mejor procedimiento para el tratamiento de un aborto incompleto (inducido ilegalmente) no ha sido definido. Tanto el curetaje como la aspiración por vacío son satisfactorios. El procedimiento dependerá de un número de variables que incluye el tiempo transcurrido desde la iniciación del aborto hasta el tratamiento, la extensión, si existe, de la infecciôn pélvica, y la etapa (amenaza, inevitable, incompleta) del aborto.

Simultáneamente con la liberización de las leyes del aborto, se han desarrollado, métodos más seguros y más eficaces para inducir el aborto. Los resultados de numerosos estudios ha indicado que las tasas merosos estudios han indicado que las tasas de complicaciones del aborto (pérdida de sangre excesiva, trauma cervical y uterino, procedimientos incompletos) aumentan con el aumento de la edad gestacional (Figura 8). En el primer trimestre del embarazo el aborto puede ser inducido con seguridad, usando aspiración por vacío o dilatación y curetaje. Parece ser que la aspiración por vacío, cuando se compara con la dilatación y el curetaje, se asocia a tasas más bajas de complicaciones precoces en el primer trimestre, a tasas similares en el último período del primer trimestre, y a tasas más altas en el segundo trimestre de embarazo.

La terminación de un embarazo sospechado dentro de los 14 días siguientes a la fecha en que se espera el período menstrual ha sido denominada con diferentes términos: aspiración endometrial, extracción menstrual, terminación atraumática de embarazo, y regulación menstrual (RM). Sin tener en cuenta al término particular utilizado,este procedimiento, que es similar al de aspiración por vacío, ha sido demostrado ser eficaz y seguro. La tasa de complicaciones con este procedimiento es más o menos de 1.8 por ciento, y cerca de 2.0 por ciento de las pacientes que han sido tenidas como embarazadas, el procedimiento no termina con el embarazo. El embarazo solo puede ser afirmado por 
una evaluación histológica del contenido uterino aspirado, ya que la mayoría de las pruebas urinarias para embarazo no son seguras antes de seis o siete semanas de gestación. La mayoría de las complicaciones reportadas son leves (vómitos, laceración cervical, fiebre, etc.) y no requieren ningún tratamiento prolongado $u$ hospitalización. La desventaja principal de la regulación menstrual ( $\mathrm{RM}$ ) es que del 25 al 45 por ciento de las pacientes no están embarazadas en el momento de la intervención. Aunque hacen falta mejores métodos de selección para reducir la proporción de pacientes no embarazadas que se someten a RM, no existe ningún método para uso general en clínica, con excepción del de aplazar el procedimiento hasta que los signos y síntomas propios de embarazo puedan establecerlo. Desafortunadamente, el postponer el procedimiento se asocia a una tasa más alta de complicaciones.

Dentro de dos semanas de un período menstrual atrasado, las pruebas inmunológicas de embarazo no son seguras y se asocian a una tasa alta de falsos negativos. Aunque se han desarrollado pruebas más exactas de embarazo, como las radioinmunológicas y técnicas de receptor que pueden detectar niveles mínimos de HCG, estos métodos son costosos y probablemente no estarán al alcance para uso general en clínica, por varios años.

Para reducir la proporción de mujeres no embarazadas que se someten a un procedimiento de $R M$, algunos investigadores han evaluado el uso de dosis altas de progesterona $\mathrm{y} / \mathrm{o}$ estrógenos, para inducir sangrado uterino. Los resultados de estos estudios indican que este tratamiento no presenta ventajas aparentes y que la manera más efectiva para reducir el número de intervenciones innecesarias es retrasar el procedimiento por una semana. Muchas mujeres que no están embarazadas comenzarán a menstruar normalmente durante este período de tiempo.

El procedimiento para terminar un embarazo sospechado hasta con 14 días de atraso menstrual, es usualmente realizado con una cánula plástica flexible de diámetro pequeño $(4,5$, o $6 \mathrm{~mm})$, conectada a una jeringa de $50 \mathrm{cc}$ que se utiliza como fuente de vacío (Figura 9). En más del 95 por ciento de las pacientes, la cánula puede ser insertada dentro del útero sin nin- guna dilatación mecánica previa del cervix. Tanto la cánula plástica como la jeringa pueden volver a usarse después de ser esterilizadas.

Se han conducido investigaciones extensivas para encontrar y evaluar métodos efi caces y seguros de terminar embarazos en el segundo trimestre (12-24 semanas menstruales de gestación). Se han evaluado varios agentes para uso intra y extra amniótico, solución salina hipertónica,

tico, tales como: solución salina hipertónica, agua destilada, urea, oxitocina, pros taglandinas $\mathrm{E}_{2}, \mathrm{~F}_{2 \mathrm{a}} \mathrm{y}$ algunos de sus análogos, alcohol, formaldehído y rivanol. Sin embargo, si se intenta hacer abortar a la paciente con un mínimo de daño psicológico y devolverla a su vida normal lo antes posible, pocos de los abortifacientes mencionados hasta ahora satisfacen estos criterios. Algunos de los abortifacientes ya mencionados, si no están suplementados con otros agentes, como los oxitócieos, están asociados con instilaciones prolongadas y subsecuentemente con tasas altas de infección. Así mismo, abortifacientes como las soluciones salinas hipertónicas, cuando se administran inapropiadamente, por vía intravascular, por ejemplo, pueden determinar muertes maternas y causar complicaciones serias como hipernatrenimia y cambios en las propiedades de coagulación de la sangre.

Aunque las prostaglandinas $\left(\mathrm{E}_{2}, \mathrm{~F}_{2 \mathrm{a}} \mathrm{y}\right.$ algunos de sus análogos), parecen ser seguras y eficaces como abortifacientes, son muy costosas, no tienen una distribución amplia y cuando se administran en las dosis necesarias para abortar, se asocian con tasas altas de efectos colaterales sistémicos (vómitos, diarrea, fiebre). En este momento se están haciendo investigaciones para evaluar vías de administración de prostaglandinas que no sean las intra o extra amnióticas. Varios estudios han demostrado que la administración intramuscular de prostaglandina y el colocar supositorios intravaginales de prostaglandina pueden inducir eficazmente un aborto en el primer y segundo trimestre. Sin embargo, todavía no parece haber ninguna ventaja en la inducción del aborto con prostaglandinas en el primer trimestre, ya que en ese período el procedimiento de aspiración por vacío puede ser realizado eficaz y seguramente. La prostaglandina $\mathrm{F}_{2}$ a ha sido usada para regulación menstrual; sin embargo, las tasas altas de efectos co- 
laterales gastrointestinales no hacen el procedimiento competitivo con la aspiración por vacío.

Se debe notar que el uso de prostaglandinas no está confinado a la inducción de aborto. También han sido utilizadas para la inducción de parto, para el tratamiento de úlceras del estómago, arterioesclerosis periférica, enfermedad congénita cianótica del corazón e hipertensión, y para inducir la ovulación en el ganado vacuno y caballar. Se espera que muchos otros usos para la prostaglandina, incluyendo su uso en la reproducción humana, se descubrirá a medida que los científicos evalúen mayormente su potencial farmacológico.

Aunque el aborto inducido (ya sea legal o ilegal) no puede y no debe ser visto por la paciente o el médico como un método anticonceptivo, es sin duda un método eficaz de control de la fecundidad. Un buen número de estudios han demostra do que las mujeres no solo aceptan consejos sobre anticonceptivos después de un aborto, sino que una gran proporción de ellas aceptan métodos de alto uso y efectividad (orales, DIUs, esterilización). Hacen falta más estudios para evaluar las tasas de continuación de uso de los anticonceptivos a largo plazo en aquellas mujeres que los aceptan después de un aborto, inducido legal o ilegalmente.

\section{COMENTARIOS}

Habrá necesidad de estudios futuros para evaluar los efectos del uso prolongado de DIU, orales, y esterilización. Todos estos métodos aparentemente son los que están al alcance en este momento. Parece que el pequeño riesgo de muerte o de complicaciones series que ocurren con el uso de DIUs, anticonceptivos orales, y esterilización, vale la pena de correrse cuando se compara con la protección que estos métodos proveen. Probablemente el riesgo de muerte en un parto a término es más alto que el riesgo de muerte con el uso de DIUs, orales, o de esterilización.
Así mismo, en países donde el abortu inducido es ilegal, la muerte, las complicaciones serias, y la esterilidad subsecuente son resultados frecuentes de los intentos de las mujeres para terminar embarazos no deseados.

La anticoncepción no puede seguir siendo respnnsabilidad única de los obstetras y los ginecólogos. El propiciar anticoncepción adecuada para todas las personas que la requieran debe ser una extensión de los programas de salud pública que financian los gobiernos y no solo de las organizaciones privadas, y deben formar oprte integral de los programas nacionales de salud

\section{RECONOCIMIENTO}

Los autores agradecen la asistencia técnica prestada por Elena Tomaro en la preparación de este trabajo.

\section{Cuadro No. 1}

TASAS DE FRACASO (EMBARAZOS POR 100/MUJER) PARA METODOS SELECCIONADOS DE ANTICONCEPCION

\begin{tabular}{|l|c|}
\hline Método & $\begin{array}{c}\text { Tasa de Fraca- } \\
\text { so durante el } \\
\text { Uso }\end{array}$ \\
\hline Ninguno & 80 \\
Ritmo (calendario) & 35 \\
Retiro & $20-25$ \\
Espuma & 30 \\
Diafragma & $20-25$ \\
Condon & $15-20$ \\
DIU & 6 \\
Orales & $2-5$ \\
Esterilización (mascul.) & 1 \\
Esterilización (femenina) & $0.1-2$ \\
\hline
\end{tabular}

Adaptado de Tecnología Anticonceptiva 1974-1975. Emory University Family Planning Program. Atlanta, Georgia. 
Cuadro No. II

TASAS DE EMBARAZOS, EXPULSION Y RETIRO AL AÑO DE DIUS SELECCIONADOS

\begin{tabular}{|c|c|c|c|c|c|}
\hline \multirow[t]{2}{*}{ DIU* } & \multicolumn{2}{|c|}{. $\quad$. } & \multicolumn{3}{|c|}{ Tasa de Eventos/100 mujeres } \\
\hline & $\begin{array}{r}\text { Primeras } \\
\text { nserciones }\end{array}$ & $\begin{array}{l}\text { Mujer-meses } \\
\text { de uso }\end{array}$ & Embarazo & Expulsion & $\begin{array}{l}\text { Retiro por } \\
\text { sangrado/dolor }\end{array}$ \\
\hline Dalkon Shield (1) & 2848 & 15102 & 2.1 & 2.5 & 10.4 \\
\hline Dalkon Shield (2) & 4191 & 33397 & 2.8 & 2.0 & 4.2 \\
\hline Lippes Loop D(3) & 4557 & $* *$ & 2.4 & 9.1 & 10.9 \\
\hline Lippes Loop D(3) & 7553 & $* *$ & 2.5 & 8.0 & 12.9 \\
\hline Lippes Loop D(3) & 883 & 8899 & 1.2 & 9.0 & 4.8 \\
\hline Lippes Loop D(4) & 882 & 6634 & 2.3 & 10.3 & 12.6 \\
\hline Sa-T-Coil 255 (5) & 1179 & 9750 & 0.4 & 19.0 & 11.0 \\
\hline $\mathrm{Cu}-\mathrm{T}-200$ & 6785 & 6727 & 0.8 & 6.0 & 14.2 \\
\hline $\mathrm{Cu}-\mathrm{T}-200$ & 472 & 4430 & 1.7 & 5.4 & 10.7 \\
\hline $\mathrm{Cu}-\mathrm{T}-300$ & 347 & 3442 & 0.6 & 6.1 & 9.2 \\
\hline $\mathrm{Cu}-7(6)$ & 400 & 2555 & 0.6 & 17.1 & 8.4 \\
\hline $\mathrm{Cu}-7$ (6) & 342 & 1197 & 1.1 & 6.7 & 3.0 \\
\hline $\mathrm{Cu}-7$ (6) & 186 & 1693 & 1.1 & 4.1 & 12.4 \\
\hline Fluid Filled Device ( 8 ) & 3) 307 & $* *$ & 1.5 & 10.5 & 10.9 \\
\hline Progestasert-65 (9) & 1145 & 8275 & 1.4 & 8.7 & 12.2 \\
\hline Spring Coil (10) & 344 & 3064 & 0.0 & 21.0 & 10.1 \\
\hline Spring Coil-200 mm ${ }^{2}$ & & & & & \\
\hline $\mathrm{Cu}(11)$ & 100 & 1132 & 0.0 & 2.0 & 3.0 \\
\hline $\begin{array}{l}\text { Spring Coil }(60 \mathrm{mg}+ \\
\text { Progesterona(11) }\end{array}$ & 100 & 1557 & 0.0 & 5.0 & 4.2 \\
\hline
\end{tabular}

* Las referencias entre paréntesis se encuentran al final del trabajo.

** Desconocido.

FIGURA 1. Spring-Loaded Clip

FIGURA 2. Banda Silástica

FIGURA 3. Aplicador de Clip

FIGURA 4. Clip Aplicado a la Trompa

FIGURA 5. Aplicador de Bandas Silásticas

FIGURA 6. Oclusión Tipo Madlener con Banda Slástica

FIGURA 7. Tanta lum-Silástico Clip

FIGURA 8. Tasa de Complicaciones por Aborto Según Duración de Amenorrea

FIGURA 9. Jeringa de 50 cc. y Cálulas Plásticas 


\section{RESUMEN}

Después de veinte años de utilización de los métodos anticonceptivos; vale la pena hacer un estudio retrospectivo de las ventajas logradas con uno u otro método, a fin de iniciar una evaluación de efectos por su uso prolongado, tanto de la utilización del DIU como de anovulatorios y y en igual forma el uso de medios quirúrgicos esterilizantes. En cuanto al riesgo de muerte o de complicaciones serias causadas por tales medios, comparativamente se observa más bajo del producido joor un parto a término. Además en países donde el aborto inducido es ilegal, la muerte, las complicaciones serias y la esterilidad subsecuente, son consecuencias frecuentes de intentos fallidos o no de mujeres con embarazos no deseados.

\section{SUMMARY}

After twenty years of using contraceptives, it is worthwhile to make a retrospective study of the advantages obtained in order to have an evaluation of long-range effects of the IUD, antiovulants as well as the chemical esterilizing agents. As far as the risk of death or serious complications as a result of the use of contraceptive methods is concern, the percentage is lower than those caused by deliveries. Furthermore, in those countries where induced abortions are ellegal, death, serious complications and subsequent sterility are frecuent consecuences of attempts of women to rid themselves of unwanted pregnancies. 